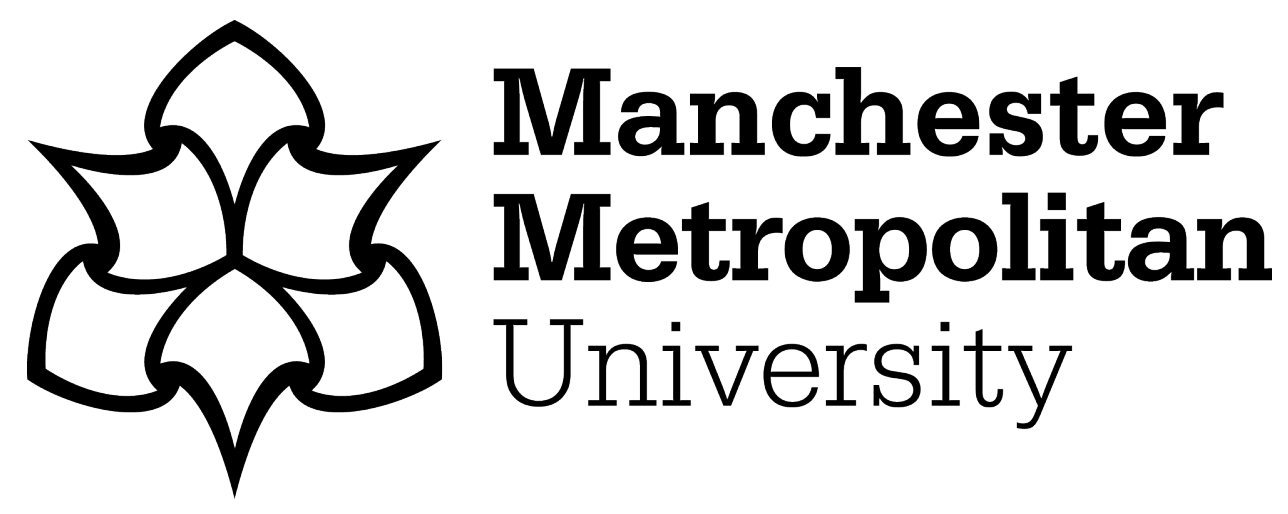

Duncan, MJ, Smith, M, Bryant, E, Eyre, E, Cook, K, Hankey, J, Tallis, J, Clarke, N and Jones, MV (2014) Effects of increasing and decreasing physiological arousal on anticipation timing performance during competition and practice. European Journal of Sport Science, 16 (1). pp. 27-35. ISSN 17461391

Downloaded from: https://e-space.mmu.ac.uk/620537/

Publisher: Taylor \& Francis

DOI: https://doi.org/10.1080/17461391.2014.979248

Please cite the published version 
RUNNING HEAD: EFFECTS OF INCREASING AND DECREASING PHYSIOLOGICAL AROUSAL

\title{
Effects of increasing and decreasing physiological arousal on anticipation timing performance during competition and practice
}

\author{
Michael J. Duncan, Mike Smith, Elizabeth Bryant, Emma Eyre, Kathryn Cook, \\ Joanne Hankey, Jason Tallis, Neil Clarke \\ Coventry University, UK
}

And

Marc V. Jones

Staffordshire University, UK

Please address correspondence and requests for reprints to Michael J. Duncan, Department of Biomolecular and Sports Sciences, Coventry University, Priory Street, Coventry, United Kingdom, CV1 5FB or email: michael.duncan@coventry.ac.uk 


\begin{abstract}
The aim of this study was to investigate if the effects of changes in physiological arousal on timing performance can be accurately predicted by the catastrophe model (Hardy 1990). Eighteen young adults (8 males, 10 females) volunteered to participate in the study following ethical approval. After familiarisation, coincidence anticipation was measured using the Bassin Anticipation Timer under 4 incremental exercise conditions: Increasing exercise intensity and low cognitive anxiety, Increasing exercise intensity and high cognitive anxiety, decreasing exercise intensity and low cognitive anxiety, decreasing exercise intensity and high cognitive anxiety. Incremental exercise was performed on a treadmill at intensities of $30 \%, 50 \% 70 \%$ and $90 \%$ heart rate reserve (HRR) respectively. Ratings of cognitive anxiety were taken at each intensity using the Mental Readiness Form 3 (MRF3) followed by performance of coincidence anticipation trials at speeds of 3 and $8 \mathrm{mph}$. Results indicated significant condition $X$ intensity interactions for $A E(P=.0001)$ and MRF cognitive anxiety intensity scores $(P=.05)$. Post-hoc analysis indicated that there were no statistically significant differences in $\mathrm{AE}$ across exercise intensities in low cognitive anxiety conditions. In high cognitive anxiety conditions, timing performance $A E$ was significantly poorer and cognitive anxiety higher at $90 \% \mathrm{HRR}$, compared to other exercise intensities. There was no difference in timing responses at $90 \% \mathrm{HRR}$ during competitive trials irrespective of whether exercise intensity was increasing or decreasing. This study suggests that anticipation timing performance is negatively affected when physiological arousal and cognitive anxiety are high.
\end{abstract}

Keywords: Exercise intensity; Bassin Anticipation Timer; Anxiety; Competition 


\section{Introduction}

Numerous research papers have documented the varying consequences of exercise intensity on cognitive, perceptual and psychomotor skills. (Davey, Thorpe, \& Williams, 2002; Lyons, Al-Nakeeb, \& Nevill, 2008; Lyons, Al-Nakeeb, \& Nevill, 2006a; Al-Nakeeb \& Lyons, 2007). The findings of such studies have been equivocal with some finding that exercise improves performance (Lyons, et al., 2006a; Lyons, Al-Nakeeb, \& Nevill, 2006b), some it decreases performance (Davey, et al., 2002) and some finding no effect (Lyons, et al., 2008; McMorris, 2000). One suggestion for the contrasting results is the failure to examine performance during exercise (e.g., Davey, et al., 2002; Lyons, et al., 2006a; Lyons, et al., 2006b; Royal, et al., 2006). This anomaly has led authors (Tomporowski, 2009; Lyons, et al., 2008) to suggest that to accurately examine the effect of exercise intensity on perceptual, motor or cognitive performance, the task should be completed while the participant is actually exercising at the intensity of interest.

The suggestion that exercise is a physiological (Audiffren, 2009; Duncan, Smith, \& Lyons, 2013), cognitive (McMorris, Sproule, Turner, \& Hale, 2011) and psychomotor (Lyons et al., 2008) stressor is well established in the literature. With performance following an inverted $U$ relationship, where moderate intensity exercise is associated with enhanced performance and higher exercise intensity is associated with poorer performance.

More recently, Duncan et al (2013) presented data pertaining to the effect of exercise intensity on coincidence anticipation timing performance (CAT) which relates to the making of interceptive actions (Poulton, 1957) and the ability to predict and coordinate a movement response to the arrival of a moving object (Payne, 1986). For example, catching a cricket ball or striking a moving ball in tennis (Sanders, 2011). Consequently understanding the effect of exercise intensity on CAT performance is of interest to psychologists, sport coaches and sports scientists amongst others. 
Duncan et al (2013) reported significantly poorer coincidence timing when exercise was undertaken at a high intensity $(90 \%$ heart rate reserve $(H R R))$ and stimulus speed was also high (8mph) compared to rest and exercise at $70 \%$ HRR. There were also no differences in anticipation timing scores across exercise intensity when stimulus speed was low (3mph). Duncan et al (2013) suggested that their results supported the Catastrophe Model's predictions (Hardy, 1990). This suggestion was based on the assertion that when physiological arousal was high (exercise intensity of $90 \% \mathrm{HRR}$ ) and stimulus speed was most cognitively demanding (stimulus speed of $8 \mathrm{mph}$ ) there was a severe reduction in timing accuracy. Although the study by Duncan et al (2013) provides some data relating to the effect of exercise intensity of timing performance, further investigation is needed as only rest, $70 \%$ and $90 \%$ HRR were used as intensities, providing limited range of physiological arousal with which to compare performance across (Duncan et al 2013).

One model that can explain how exercise may influence performance is the catastrophe model (Hardy \& Parfitt, 1991). The model's predictions are based on the tenet that there is a cognitive and physiological component that interacts with each other during performance. Specifically, the model predicts that when cognitive anxiety is low, the relationship between physiological arousal and performance should follow an inverted-U. Whilst when cognitive anxiety is high a hysteresis will occur and performance will follow a different path dependant on whether physiological arousal is increasing or decreasing (Jones \& Hardy 1997).

There are several researchers who have tested the predictions of the catastrophe model (Hardy, Beattie, \& Woodman, 2007; Hardy, Woodman, \& Carrington, 2004; Edwards \& Hardy, 1996; Hardy, Parfitt, \& Pates, 1994; Krane, Joyce, \& Rafeld, 1994; Hardy \& Parfitt, 1991). For example, Hardy and Parfitt (1991) increased or decreased the physiological arousal of eight female basketball players, before attempting a basketball free throw shot. Decrements in performance were worst when cognitive anxiety and physiological arousal was at its highest, with the investigators concluding that the catastrophe model was an accurate predictor of how changes in physiological arousal, cognitive anxiety and somatic 
anxiety can affect performance. There are limitations to a number of studies (Edwards \& Hardy, 1996; Hardy \& Parfitt, 1991; Hardy, Parfitt, \& Pates, 1994) in that a time to event paradigm was used. Specifically, cognitive and somatic anxiety was measured by self-report (i.e. Competitive State Anxiety Inventory-2 (CSAI-2), Marten, Burton, Vealey, Bump, \& Smith, 1990) prior to the performance as Hardy \& Parfitt, (1991) suggest "to manipulate cognitive anxiety independently of physiological arousal" (p.168). While, Krane, Joyce, \& Rafeld (1994) used the Mental Readiness Form (MRF; Murphy, Greenspan, Jowdy, \& Tammen, 1989) at a softball tournament where participants were required to complete the MRF before entering the batter's box, which was as close to performance as possible (Krane, Joyce, \& Rafeld, 1994). We argue that a more accurate way to examine the catastrophe model is to measure physiological arousal and cognitive anxiety in situ.

The aim of the present study is to examine if the effects of 'in-event' changes in physiological arousal and cognitive anxiety on task performance can be accurately predicted by the catastrophe model. The study hypothesised that as a consequence of manipulating exercise intensity a task performance 'catastrophe' will occur when physiological arousal is high and cognitive anxiety is elevated, when compared to resting values. Secondly, the study hypothesised that a hysteresis effect on task performance will occur depending whether physiological arousal was increasing or decreasing.

\section{Methods}

\section{Participants}

Following institutional ethics approval and informed consent, 18 physically active adults (8 males, 10 females, mean age $=23.6 \pm 4.2$ years) volunteered to participate in the study. Participants were drawn from the institution's sport and exercise science programme, were regularly engaged in competitive sport activities and reported being in health. 


\section{Performance Measures}

Coincidence Anticipation Timing (CAT)

The Bassin Anticipation Timer (Model 35575, Lafayette, USA) was used to assess coincidence anticipation timing in the present study because it is the most widely validated measure of coincidence anticipation currently available (Sanders, 2011; Diggles-Buckles \& Bassin, 1990). So that participants could complete the CAT trials whilst running, the CAT (2.24m in length) was set up horizontally across the front of the treadmill with the LED lights facing the participant. None of the lights on the runway were blanked and the target light was light \#13. The sequentially lighted LED lamps illuminate in a linear pattern with movement occurring from right to left. The mean time to complete each CAT trial was 45 seconds. Scores were recorded in milliseconds and whether the response was early or late. The start and end speeds remained constant at 3 and 8 miles $^{-1}$ for all trials. Start and end speeds were counterbalanced within trials. To reduce the likelihood that the participant could internally time the trial, cue delay (visual warning system) was set as random on the timer with a minimum delay of 1 second and a maximum delay of 2 seconds. For each trial, the signal was initiated by the experimenter, with the participant being asked to press a trigger button, with their dominant hand, as close to the arrival time of the stimulus at the target location as possible.

Heart Rate and Rating of Perceived Exertion

Heart rate was monitored continuously throughout each experimental trial via a Polar RS400 heart rate monitor (Polar OY, Kuopio, Finland). The Borg (1970) 6-20 rating of perceived exertion (RPE) scale was also used as a measure of exercise exertion during experimental trials.

Cognitive Anxiety 
Cognitive state anxiety was measured during performance by using the Mental Readiness Form 3 (MRF-3, Krane, 1994). The MRF-3 has three, bipolar; 11-point Likert scales that are anchored between worried-not worried for the cognitive anxiety scale, tense-not tense for the somatic anxiety scale, and confident-not confident for the self-confidence scale. The MRF-3 is a shorter and more expedient alternative to the Competitive State Anxiety Inventory-2 (CSAI-2; Martens, et al., 1990) and Krane's validation work revealed correlations between the MRF-3 and the CSAI-2 subscales of .76 for cognitive anxiety, .69 for somatic anxiety and .68 for self- confidence (Krane, 1994).

\section{Procedures}

The study used a within-subjects, repeated measures design and required five visits to the institution's human performance laboratory. All trials occurred at the same time of day for each participant to minimise any impact of circadian variation on performance. The first visit comprised a familiarisation period with the CAT, where participants were given 30 attempts at each of the stimulus speeds used in the study ( 3 and $8 \mathrm{mph})$, and establishment of resting heart rate. Resting heart rate (HRrest) was also obtained from each participant by asking them to lie down in a prone position for 15 minutes whilst wearing a heart rate monitor (Polar RS400, Polar Electro Oy, Kempele, Finland), in a quiet room void of visual or auditory distractions. Maximum heart rate (HRmax) was estimated at 220 minus the participant's age. Both HRrest and HRmax were then recorded and used to calculate $30 \%, 50 \% 70 \%$ and $90 \%$ heart rate reserve (HRR) (Karvonen, Kentala, \& Mustala, 1957).

On completion of the baseline session, participants then undertook 4 incremental exercise trials. These comprised two trials (increasing physiological arousal and decreasing physiological arousal) in two conditions; perceived competition situation (high cognitive anxiety) and perceived practice situation (low cognitive anxiety), necessitating 4 trials. In every trial participants responded to stimuli at $3 \mathrm{mph}$ (low cognitive load) and $8 \mathrm{mph}$ (high 
cognitive load). Experimental trials were presented in a counterbalanced order and comprised of the following:

- Incremental exercise increasing in intensity in a perceived competitive situation

- Incremental exercise increasing in intensity in a perceived practice situation

- Incremental exercise decreasing in intensity in a perceived competitive situation

- Incremental exercise decreasing in intensity in a perceived practice situation

In each trial, coincidence anticipation and MRF-3 scores were taken at rest and at $30 \%$, $50 \%, 70 \%$ and $90 \%$ HRR. An incremental running protocol on a motorised treadmill (HP Cosmos Ltd, Germany) was used to induce exercise arousal states. All trials began with a warm up based at $2-3 \mathrm{mph}$. In trials where exercise intensity was increasing, the workload was then increased by $1 \mathrm{mph}$ every $30 / 60$ s until the participant reached the desired intensity as determined by $30 \%, 50 \%, 70 \%$ and $90 \%$ of heart rate reserve (HRR) (Karvonen et al., 1957). In trials where exercise began at $90 \%$ HRR and decreased in intensity, the treadmill speed was increased from the warm-up speed to the $90 \%$ HRR value within a period of 120s. The workload was then decreased by $1 \mathrm{mph}$ every $30 / 60$ s until the participant reached the desired intensity. Throughout the test procedures, heart rate was monitored using heart rate monitors. Borg's (1970) rating of perceived exertion (RPE) scale was used as an adjunct to the monitoring of heart rate. Participants were required to achieve an RPE of 6 for pre-trial (rest) condition, $12-13$ for the $30 \% \mathrm{HRR}$ condition, $14-15$ for the $50 \% \mathrm{HRR}$ condition, 16-17 for the $70 \%$ HRR condition and $18-19$ for the $90 \%$ HRR exercise condition. Once the desired intensity was reached, as determined by both measures (\%HRR and RPE) simultaneously, participants were then required to maintain this intensity for a further two minutes to ensure that participants were truly at the desired steady-state intensity. At which point, participants performed 10 trials on the coincidence anticipation task at a stimulus speed of $3 \mathrm{mph}$ (Low Cognitive demand) and 8mph (High Cognitive demand) whilst still running. Presentation of stimulus speeds was counterbalanced. 
The total time to complete each experimental trial was similar irrespective of whether exercise intensity was increasing or decreasing and comprised approximately 20 minutes of running, with the whole trial protocol taking approximately 30 minutes per participant, per trial.

\section{Manipulation of cognitive anxiety}

To manipulate cognitive anxiety across the trials standardised instructions lasting approximately 1-minute were employed before the start of each experimental trial. This methodology has been used in prior research as a stressor to elicit increases in cognitive anxiety (Barker, Jones, \& Greenlees, 2010; Hardy, Parfitt, \& Pates, 1994; Turner, Jones, Sheffield, \& Cross, 2012). The statements comprised of demand appraisals in the line with the biopsychosocial model of challenge and threat which informed participants that the performance [CAT] scores indicated cognitive ability and that they would be required to complete 20 anticipation timing trials at each exercise intensity (10 trials at a slow and 10 trials at a fast speed). These were considered as the high cognitive anxiety trials, in line with Barker et al. (2012). Participants were also informed that two of the trials (one increasing exercise intensity and one decreasing exercise intensity) were considered practice trials and would only be used to examine the consistency of their own performance but would not be used further and that the other two trials were considered as competitive. These were considered as the low cognitive anxiety trials, in line with Barker et al. (2012). In the case of competitive trials, participants were told their CAT scores would be compared to all other participants and publically posted in ranking order, and that they would need to try very hard to perform well. Participants were then asked to stand for one minute before the trial began

\section{Statistical Analysis}

The results are expressed as mean and standard error (S.E.). For CAT scores each participant's raw scores across each of the stimulus speeds were summarised into a score for absolute error $[\mathrm{AE}]$. AE represents the absolute value of each raw score disregarding 
whether the response was early or late and is the most commonly reported measure of timing error in the coincidence timing literature (Sanders, 2011). Data for CAT was analysed using a 2 (stimulus speed) $\times 4$ (condition) $\times 5$ (intensity) $\times 2$ (gender) ways repeated measures analysis of variance (ANOVA). Two (one for each stimulus speed), 4 (condition) X 5 (intensity) X 2 (gender) ways repeated measures ANOVAs were then used as follow up tests to confirm the results from the $2 \times 4 \times 5 \times 2$ ways ANOVA. Data for MRF- 3 scores was analysed using a 4 (condition) X 5 (intensity) $\times 2$ (gender) repeated measures ANOVA In all cases backwards elimination to achieve a parsimonious solution was employed. Where significant differences were found, Bonferroni post-hoc pairwise comparisons were used to determine where the differences lay. When Bonferroni adjustments were made an alpha level of $P=.025$ was used to account for family-wise error rate. The truncated product method (Zaykin, Zhivotovsky, Westfall, and Weir, 2002) was used to combine all the $P$ values in this study to determine whether there was a bias from multiple comparison testing. The truncated product method $P$ value was $<0.0001$, indicating that the results are not biased by multiple comparisons. Partial eta squared $\left(\eta^{2}\right)$ was also used as a measure of effect size. The Statistical Package for Social Sciences (SPSS, Version 20, Chicago, II, USA) was used for all analysis and statistical significance was set, a priori, at $p=0.05$.

\section{Results}

\section{Coincidence Anticipation timing}

Using a 2 (stimulus speed) $\times 4$ (condition) X 5 (intensity) X 2 (gender) ways repeated measures ANOVA, results indicated a significant speed $X$ condition $X$ intensity interaction $(P$ $=.05$, Partial $\eta^{2}=.095$ ). Data were then re analysed using two (one for each stimulus speed), 4 (condition) $\times 5$ (intensity) $\times 2$ (gender) ways repeated measures ANOVAS as follow up analysis. Data for Absolute Error (AE) at 3mph indicated a significant main effect for exercise intensity $\left(F 4,68=3.371, P=.014\right.$, Partial $\left.\eta^{2}=.165\right)$. Scores for AE were significantly better at $70 \% \mathrm{HRR}(\mathrm{P}=.001)$ compared to $90 \% \mathrm{HRR}$. Scores for $\mathrm{AE}$ at 30\%HRR $(P=.014), 50 \%$ HRR $(P=.005)$ compared to $90 \%$ HRR did not meet the alpha 
value set for significance when considering the Bonferroni adjustment. Mean $\pm S E$ of $A E$ scores across exercise intensities for each condition are presented in Figure 1.

Figure 1 Here

When the same analysis (i.e., a 4 (condition) $\times 5$ (intensity) $\times 2$ (gender) repeated measures ANOVA) was conducted with coincidence anticipation data at a stimulus speed of 8mph there was a significant condition $X$ intensity interaction for $A E(F 12,192=3.603, P=$ .0001 , Partial $\eta^{2}=.184$, See Figure 2). Post-Hoc analysis indicated no significant difference between $A E$ in increasing intensity and decreasing intensity competition conditions $(P=.08)$ or increasing and decreasing practice conditions $(P=.08)$. AE was significantly different between increasing intensity, competition and increasing intensity practice conditions when exercise intensity was at $90 \% \mathrm{HRR}(\mathrm{P}=.001)$. AE was also significantly different when exercise intensity was at $90 \% \mathrm{HRR}$ between decreasing intensity competition and decreasing intensity practice $(P=.003)$ conditions. Additionally, $A E$ at $90 \% \mathrm{HRR}$ was different between increasing intensity, competition and decreasing intensity, practice $(P=.007)$ and between decreasing intensity, competition and increasing intensity, practice $(P=.004)$. Only the latter was significant when adjusting for Bonferroni corrections.

Figure 2 here

MRF-3: Cognitive Anxiety Intensity

Results from a 4 (condition) X 5 (intensity) X 2 (gender) repeated measures ANOVA indicated a significant condition $X$ time interaction for cognitive anxiety $(F 12,192=12.778$, 
$P=.002$, Partial $\eta^{2}=.148$, See Figure 3). Post-Hoc analysis indicated that at $30 \% \mathrm{HRR}$, cognitive anxiety scores were higher in the increasing intensity, competition condition compared to both the decreasing intensity, competition $(P=.034)$ and decreasing intensity, practice $(P=.007)$ conditions but did not meet the adjusted alpha value required to be significant when using Bonferroni adjustments. Cognitive anxiety scores were significantly higher in the increasing intensity competition condition compared to the decreasing intensity practice $(P=.002)$ condition at $50 \%$ HRR. There were no significant differences across conditions at $70 \% \mathrm{HRR}$ (all $\mathrm{P}>.05$ ). At an intensity of $90 \% \mathrm{HRR}$ there were no significant differences between the increasing intensity, competition and decreasing intensity, competition $(P=.261)$ conditions or the increasing intensity, practice and decreasing intensity, practice $(P=.794)$ conditions. Cognitive anxiety scores were higher in the increasing intensity, competition condition compared to the increasing intensity, practice $(P=$ .013) and the decreasing intensity, competition condition compared to the decreasing intensity, practice condition $(P=.012)$. In these instances alpha values did not reach the Bonferroni adjusted level for statistical significance. There was a significant difference between increasing intensity competition and decreasing intensity practice conditions $(P=$ $.001)$.

Figure 3 Here

\section{Discussion}

This study builds on previous work by (Edwards \& Hardy, 1996; Hardy \& Parfitt, 1991; Hardy, Parfitt, \& Pates, 1994; Krane, Joyce, \& Rafeld, 1994) who measured 'pre-event' anxiety rather than 'in-event' anxiety when investigating the catastrophe model's predictions in relation to task performance. Results partially support the predictions of the catastrophe model in several ways. For example, when cognitive anxiety is low the effect of exercise 
intensity on CAT performance follows an inverted-U relationship, where performance is poorer at rest up until $70 \% \mathrm{HHR}$ (see Figure 1) when the CAT stimulus speed is slow (i.e. $3 \mathrm{mph}$ ). The change in CAT performance reported here was irrespective of practice or competition conditions and whether exercise intensity was increasing or decreasing. When the CAT stimulus speed is at $8 \mathrm{mph}$, there is a significant increase in timing error (i.e., poorer performance) evident during high intensity exercise in the competitive trials irrespective of whether exercise intensity is increasing or decreasing.

In this respect, the first hypothesis which is that a performance 'catastrophe' would occur when physiological arousal was high at the same time cognitive anxiety and somatic anxiety were elevated compared to resting values, is at least partially supported. It could be argued that the changes in CAT performance are not indicative of a 'catastrophe' per se, potentially due to the way in which cognitive anxiety was manipulated, which has been previously been reported as limiting factor by Hardy et al., (2007). In the current study, selfreported cognitive anxiety was assessed and while cognitive anxiety was 'higher' in competitive conditions compared to practice conditions, the highest scores were actually midway on the MRF-3 scale. It is possible that the method of anxiety manipulation employed in the present study was not successful in creating 'high' anxiety in participants. Practically and ethically, it is difficult to create conditions where anxiety would be elevated to levels at the higher end of the MRF-3 scale. Prior meta-analysis by Craft, Magyar, Becker, \& Feltz, (2003) and, Woodman, and Hardy, (2003) concluded that self-reported anxiety is not strongly related to performance outcomes. It may be that self-report measures in the current study may not be effective in accurately assessing cognitive anxiety and, hence explain the moderate levels of reported anxiety found in the present study. Cognitive anxiety can also be perceived as debilitative or facilitative (Burton 1988) to performance irrespective of how low or high the intensity scale is reported. Although the direction (i.e. facilitative or debilitative) of the anxiety response was not included in the present study it would be a useful consideration for future research. 
The current study assessed cognitive anxiety during performance trials (rather than before performance trials) unlike the majority of prior work on this topic. Some studies have however attempted to assess anxiety in situ. For example, Hardy et al. (2004) who measured self-confidence, cognitive anxiety and somatic anxiety prior to each golfing shot. Despite this, the pre situ measurement, even immediately before a golfing shot as in Hardy et al. (2004) may not be directly comparable to the in situ measurement in the present study given the different physiological arousal likely to occur during high intensity aerobic exercise as compared to golf.

Therefore, the results of the present study support the catastrophe model in that timing performance was negatively affected when physiological arousal was high (via manipulation of exercise intensity), and cognitive anxiety was higher than at rest (via the manipulation of competition/practice climate). The results of the study would seem to suggest that cognitive anxiety was the splitting factor in whether there was a 'catastrophic' failure in performance but only when the performance task was more cognitively demanding (via a higher stimulus speed on the anticipation timer). Such data aligns with prior assertions by Hardy and Parfitt (1991) and Krane et al (1994). Likewise, when the less cognitively demanding stimulus speed was considered the results support claims made by McMorris et al (2011) that there is an inverted- $U$ relationship between exercise intensity and performance. In addition, a major prediction of the catastrophe model is that when cognitive anxiety is high, performance will follow a different path depending whether physiological arousal is decreasing or increasing, which has been termed 'hysteresis' (Hardy, 1990). The present study also hypothesised that a hysteresis effect would be evident where timing performance would differ depending whether physiological arousal was increasing or decreasing. There was no evidence of hysteresis effects in the present study and as such this aforementioned hypothesis was not supported. The lack of hysteresis effect in the present study may be due to a number of factors including the use of increasing and 
decreasing (i.e. physiological arousal and cognitive anxiety) trials on different days and the reduction in cumulative fatigue which would accompany such protocols.

Moreover, to the authors' knowledge and based on the 'in situ' nature of the method, the current study is an improvement on previous investigations of the catastrophe model to date, and consequently one which adds to the extant literature in the area. Although the catastrophe model has shown promise in explaining the relational effect of anxiety on performance (Hardy \& Parfitt, 1991), due to the difficulty in testing the predictions of the model, very few studies have been completed in the last 25 years. We add to the previous literature in the area by testing anxiety 'in-event' and prior to performance during exercise. Therefore, although the results of the present study add support to the catastrophe model and prior research in the area (Hardy \& Parfitt, 1991; Krane et al., 1994), the use of an 'inevent' task in the present study extends the topic area in a way which has previously not been forthcoming. We also acknowledge that the method used in the present study differs from previous studies of the catastrophe model as the increasing and decreasing exercise intensity conditions were conducted on different days. This method is unlike other studies (e.g., Hardy \& Parfitt, 1991) that used protocols that increase and then decrease in arousal during the same experimental session. From the context of exercise induced physiological arousal such protocols are problematic. The process of increasing exercise intensity and then immediately decreasing exercise intensity may not solely represent decreasing exercise induced physiological arousal but rather will include a cumulative fatigue aspect from the previous increases in exercise intensity. The protocol employed in the present study removes the possibility that cumulative fatigue from an increasing exercise induced arousal condition would impact on performance in the decreasing exercise induced arousal condition.

The results of the present study are novel as physiological arousal and cognitive anxiety was assessed 'in situ' alongside a measure of performance. In this respect, the results suggest that cognitive anxiety was significantly higher (compared to rest) during high 
intensity exercise $(90 \% \mathrm{HRR})$ during both the competitive performance conditions compared to the practice performance conditions, which addresses key criticisms of past research study design, namely the assessment of performance during exercise, rather than on exercise cessation (Tomporowski, 2009; Lyons, et al., 2008).

Despite the findings presented here, the present study does have some limitations. By assessing CAT during exercise we sought to build on prior recommendations (Lyons et al., 2008) that CAT should be assessed at different stimulus speeds and during rather than post exercise. In addition, allocation of treatment could not be completely blinded from participants as they were explicitly informed which trials were competition and practice trials and the nature of exercise in terms of increasing or decreasing exercise intensity was evident from the outset of each trial. Furthermore, only state anxiety was assessed in the present study. It could be argued that assessment of trait anxiety would also have added to the current study. For example, processing efficiency theory (Eysenck \& Calvo, 1992) predicts that state anxiety experienced by a performer is determined interactively by trait anxiety and the perceived threat in the performance setting, which was supported in a study by Hardy and his colleagues, who used trait anxiety quite successfully to manipulate state anxiety (Hardy et al. 2007).

Thus, in the context of the present study understanding how trait anxiety may have interacted with the perception of competition or practice may have provided valuable insight into the mechanisms by which performance catastrophes are derived. Future researchers should therefore consider the inclusion of both state and trait measures of anxiety in their designs. Finally, only cognitive anxiety was manipulated in the current study. Future research which also manipulated somatic anxiety and self-confidence would be desirable in developing scientific understanding of the catastrophe model. The time and labour intense nature of such a study prohibited the manipulation of somatic anxiety and self-confidence alongside the manipulation of cognitive anxiety and exercise induced arousal in the present research. 
Conclusions

The current study suggests that CAT performance is negatively affected when physiological arousal is high and cognitive anxiety is increased from resting values. Such effects are not seen when cognitive anxiety is lower, thus cognitive anxiety may be considered the decisive factor in predicting decrements in performance. Furthermore, it is the participant's perception of competition over practice that is a major influencing factor in increasing cognitive and somatic anxiety. Consequently, these results strongly support the predictions of the catastrophe model.

\section{References}

Al-Nakeeb, Y., \& Lyons, M. (2007). Performance of tasks with high demands on anticipation and concentration under various conditions of physical exertion. In: Proceedings of the 12th Annual Congress of the European College of Sport Science, Jyvaskyla, Finland.

Audiffren, M. (2009). Acute exercise and psychological functions: a cognitive-energetic approach. In T. McMorris, P. Tomporowski, \& M. Audiffren (Eds.), Exercise and cognitive function (pp. 340). London: Wiley-Blackwell.

Barker, J.B., Jones, M.V. \&, Greenlees, I. (2010). Assessing the immediate and maintained effects of hypnosis on self-efficacy and soccer wall-volley performance. Journal of Sport \& Exercise Psychology, 32, 243-252.

Brady, F. (1996). Anticipation of coincidence, gender and sports classification. Perceptual and Motor Skills, 82, 227-239.

Burton, D. (1988). Do anxious swimmers swim slower? Re-examining the elusive anxiety performance relationship. Journal of Sport \& Exercise Psychology, 10, 45-61.

Borg, G. (1970). Perceived exertion as an indicator of somatic stress. Scandinavian Journal of Rehabilitation Medicine, 2, 92-98. 
Craft, L. L., Magyar, T. M., Becker, B. J., \& Feltz, D. L. (2003). The relationship between the competitive state anxiety inventory-2 and sport performance: A meta-analysis. Journal of Sport \& Exercise Psychology, 25, 44-65.

Davey, P.R., Thorpe, R.D. \& Williams, C. (2002). Fatigue decreases skilled tennis performance. Journal of Sports Sciences, 20, 311-318.

Diggles-Buckles, V., \& Bassin, S. L. (1990). Knowledge of results, practice, and sex: anticipation of coincidence revisited. Perceptual and Motor Skills, 70, 131-141.

Duncan, M., Smith, M., \& Lyons, M. (2013). The effect of exercise intensity on coincidence anticipation performance at different stimulus speeds. European Journal of Sports Science. $13,559-566$.

Edwards, T., \& Hardy, L. (1996). The interactive effects of intensity and direction of cognitive and somatic anxiety, and self-confidence upon performance. Journal of Sport \& Exercise Psychology, 18, 296-312.

Eysenck, M.W., \& Calvo, M. (1992). Anxiety and performance: The processing efficiency theory. Cognition and Emotion, 6,409-434.

Gill, D.L. (1994). A sport and exercise psychology perspective on stress. Quest, 44,20-27.

Hardy, L. (1990). A catastrophe model of performance in sport. In J.G. Jones \& L. Hardy (Eds.), Stress and performance in sport (pp. 81-106). Chichester, NY: John Wiley.

Hardy, L (1996). Testing the predictions of the cusp catastrophe model of anxiety and performance. The Sport Psychologist, 10, 140-156.

Hardy, L., Woodman, T., \& Carrington, S. (2004). Is self-confidence a bias factor in higher order catastrophe models? An exploratory analysis. Journal of Sport \& Exercise Psychology, 26, 359-368.

Hardy, L., Beattie, S., \& Woodman, T. (2007). Anxiety induced performance catastrophes: investigating effort required as an asymmetry factor. British Journal of Psychology, 98, 1531.

Hardy, L. \& Parfitt, G. (1991). A catastrophe model of anxiety and performance. British Journal of Psychology, 82, 163-178. 
Hardy, L. Parfitt, G. \& Pates, J. (1994) Performance catastrophes in sport: A test of the hysteresis hypothesis. Journal of Sports Sciences, 4, 327-334.

Isaacs, L. D., \& Pohlman, R. L. (1991). Effects of exercise intensity on an accompanying timing task. Journal of Human Movement Studies, 20, 123-131.

Jones, G. \& Hardy, L. (1997). Stress and performance in sport. John Wiley \& Sons Ltd. Chichester, England.

Karvonen, M. J., Kentala, E., \& Mustala, O. (1957). The effects of training heart rate: a longitudinal study. Annales Medicinae Experimentalis et Biologiae Fenniae, 35, 307-315.

Krane, V. (1994). The Mental Readiness Form as a Measure of Competitive State Anxiety. The Sport Psychologist, 8, 189-202

Krane, V., Joyce, D., \& Rafeld, J. (1994). Competitive anxiety, situation criticality, and softball performance. The Sport Psychologist, 8, 58-72.

Lyons, M., Al-Nakeeb, Y., \& Nevill, A. (2008). The effect of moderate and high intensity fatigue on coincidence anticipation in expert and novice Gaelic games players. European Journal of Sport Science, 8, 205-216.

Lyons, M., Al-Nakeeb, Y., \& Nevill, A. (2006a). Performance of soccer passing skills under moderate and high-intensity localised muscle fatigue. Journal of Strength and Conditioning Research, 20, 197-202.

Lyons, M., Al-Nakeeb, Y., \& Nevill, A. (2006b). The impact of moderate and high intensity total body fatigue on passing accuracy of experienced and novice basketball players. Journal of Sport Science and Medicine, 5, 215-227.

Martens, R., Burton, D., Vealey, R.S., Bump, L.A., \& Smith, D.E. (1990). Development and validation of the Competitive State Anxiety Inventory-2. In R. Martens, R.S. Vealey, \& D. Burton, Competitive anxiety in sport (pp. 117-1 18). Champaign, IL: Human Kinetics.

McMorris, T., Sproule, J., Turner, A., \& Hale, B. J. (2011) Acute, intermediate intensity exercise, and speed and accuracy in working memory tasks: A meta-analytical comparison of effects. Physiology and Behavior, 102, 421-428. 
McMorris, T., Gibbs, C., Palmer, J., Payne, A., \& Torpey, N. (1994). Exercise and performance of a motor skill. Research Supplement, 15, 23-27.

Murphy, S., Greenspan, M., Jowdy, D., \& Tammen, V. (1989, October). Development of a brief rating instrument of competitive anxiety: Comparison with the CSAI-2. Paper presented at the meeting of the Association for the Advancement of Applied Sport Psychology, Seattle, WA.

Payne, V. G. (1986). The effects of stimulus runway length on coincidence anticipation timing performance. Journal of Human Movement Studies, 12, 289-295.

Poulton, E. C. (1957). On prediction in skilled movements. Psychological Bulletin, 54, 467-478.

Royal, K.A., Farrow, D., Mujika, I., Halson, S.L., Pyne, D. \& Abernethy, B. (2006). The effects of fatigue on decision making and shooting skill performance in water polo players. Journal of Sports Sciences, 24, 807-815.

Sanders, G. (2011). Sex differences in coincidence anticipation timing (CAT): A review. Perceptual and Motor Skills, 112, 61-90.

Tomporowski, P. (2009). Methodological issues: research approaches, research design, and task selection. In T. McMorris, P. Tomporowski, \& M. Audiffren (Eds.), Exercise and cognitive function (pp. 99-114). London: Wiley-Blackwell.

Turner, M., Jones, M. V., Sheffield, D. C. \& Cross, S.L. (2012) Cardiovascular indices of challenge and threat states predict competitive performance. International Journal of Psychophysiology, 86, 48-57.

Woodman, T., \& Hardy, L. (2003). The relative impact of cognitive anxiety and self-confidence upon sport performance: a meta-analysis. Journal of Sports Sciences, 21, 443-457.

Zaykin, D.V., Zhivotovsky, L.A., Westfall, P.H., \& Weir, B.S. (2002). Truncated product method for combining P-values. Genetic Epidemiology, 22, 170-185. 
List of Figures

Figure 1. Mean (+/- SE) of absolute error (msec) across conditions and exercise intensities with a stimulus speed of $3 \mathrm{mph}$.

Figure 2. Mean (+/- SE) of absolute error (msec) across conditions and exercise intensities with a stimulus speed of $8 \mathrm{mph}$.

Figure 3. Mean (+/- SE) of MRF-3 scores for Cognitive Anxiety intensity across conditions and exercise intensities. 